\section{Perfil metabólico e antropométrico de índios Aruák: Mehináku, Waurá e Yawalapití, Alto Xingu, Brasil Central, 2000/2002}

\author{
Metabolic and anthropometric profile of Aruák \\ Indians: Mehináku, Waurá and Yawalapití in the \\ Upper Xingu, Central Brazil, 2000-2002
}

\author{
Suely Godoy Agostinho Gimeno 1 \\ Douglas Rodrigues 1 \\ Heloisa Pagliaro 1 \\ Eduardo Nozaki Cano 1 \\ Evandro Emilio de Souza Lima 1 \\ Roberto Geraldo Baruzzi ${ }^{1}$
}

\section{Introdução}

A saúde de um indivíduo está intimamente relacionada ao ambiente e às condições de vida da comunidade em que ele vive. Resultados de diferentes estudos mostram que pessoas submetidas ao processo de "ocidentalização", particularmente relacionado a mudanças no estilo de vida e no hábito alimentar, tornam-se mais susceptíveis às doenças crônicas não-transmissíveis, tais com as doenças cardiovasculares e o diabetes mellitus 1,2,3. Cardoso et al. 4 comentam que os estudos feitos com povos indígenas mostram um aumento na porcentagem de indivíduos com doenças cardiovasculares, diabetes mellitus e transtornos mentais em função do processo de crise de identidade e de mudanças de hábitos.

Altas prevalências de excesso de peso e outros fatores de risco cardiovasculares, como a hipertensão arterial e o diabetes mellitus foram observados entre populações indígenas, tais como os índios Pima, que vivem no Estado do Arizona, Estados Unidos. Mudanças no estilo de vida tradicional incluindo menor grau de atividade física, consumo de dietas ricas em carboidratos, em gorduras e pobres em fibras e, conseqüentemente, a obesidade, estão associadas ao aumento tanto da incidência quanto da prevalência dessas doenças 2,5.

Populações indígenas no Brasil sofreram, nas últimas décadas, processo de mudanças no esti- 
lo de vida, mas poucas informações sobre a freqüência de fatores de risco cardiovascular estão disponíveis na literatura. Em 1964, Pazzanese et al. ${ }^{6}$ não encontram casos de arteriosclerose em índios do Parque Indígena do Xingu, no Brasil Central. Esses autores, ao examinarem 53 indivíduos de ambos os sexos e com idade presumida de 30 anos ou mais, observaram que apesar dos níveis séricos médios de lipoproteínas terem sido maiores entre as mulheres quando comparadas aos homens, estes foram sempre inferiores aos registrados para a população brasileira. $\mathrm{Na}$ ocasião, os autores atribuíram tal fato, principalmente, ao tipo de alimentação desses indígenas, baseada em grande parte na mandioca e outros tubérculos, peixe, amendoim, milho, mel, banana e outras frutas silvestres. Algumas décadas mais tarde, alguns casos de diabetes mellitus foram relatados por Vieira-Filho et al. 7 entre índios Karipúna e Palikúr, residentes no Estado do Amapá. Nascimento et al. 8, no final da década de 90, identificaram alguns casos de hipertensão arterial e de obesidade entre índios da tribo Tembé, no nordeste do Estado do Pará. Mais recentemente, em índios Guaraní-Mbyá do Estado do Rio de Janeiro, Cardoso et al. 4 encontraram prevalências de 4,8\% de hipertensão arterial, 26,7\% de sobrepeso, $4,8 \%$ de obesidade, $2,8 \%$ de hipercolesterolemia e $12,6 \%$ de hipertrigliceridemia.

O estudo INTERSALT foi um projeto de âmbito mundial desenvolvido com o objetivo de determinar a relação entre a pressão arterial e o consumo de sal (avaliado pela eliminação de eletrólitos na urina de 24 horas), executado em meados da década de 1980, e incluiu 52 povos de 32 países. Nessa investigação, quatro povos apresentaram resultados divergentes dos demais, ou seja, baixos níveis de pressão arterial, ausência ou raros casos de hipertensão e estabilidade dos níveis pressóricos com o avançar da idade. Esses quatro povos foram os Papua da Nova Guiné, um grupo nativo do Quênia, os Yanomámi da fronteira do Brasil com a Venezuela e os índios do Parque Indígena do Xingu 9.

O objetivo deste trabalho foi descrever o perfil metabólico e antropométrico de índios Aruák (Mehináku, Waurá e Yawalapití) que habitam o Alto Xingu, com ênfase nas prevalências de excesso de peso (sobrepeso e obesidade), hipertensão arterial, dislipidemias e intolerância à glicose.

\section{Métodos}

Os Mehináku, Waurá e Yawalapití, juntamente com os Kalapálo, Kuikúru, Matipú e Nahukwá, de língua Karíib, os Awetí e Kamayurá, de língua Tupi e os Trumái, de língua isolada, pertencem à cultura do Alto Xingu (região dos formadores do rio Xingu, Mato Grosso) ou do Uluri (pequeno cinto pubiano usado pelas mulheres). Os povos do Alto Xingu foram pela primeira vez descritos por Karl von den Steinen, etnólogo alemão que esteve na região em duas expedições: em 1884 e 1887 10. A convergência ou amalgamação cultural que resultou na cultura do Alto Xingu seria decorrente da longa ocupação de uma mesma área geográfica e da freqüência de casamentos intertribais.

A população Aruák, em julho do ano de 2000, era composta de 770 indivíduos de ambos os sexos (28,7\% Mehináku, 43,9\% Waurá e 27,4\% Yawalapití) dos quais 224 tinham idade de 20 anos ou mais. Para a realização deste estudo dispõe-se de informações de 201 indivíduos adultos (89,7\% do total), sendo 61 índios Mehináku (30,3\%), 81 Waurá (40,3\%) e 59 Yawalapití (29,4\%).

Os exames físico, clínico e a coleta de material biológico foram realizados em julho de 2000 na aldeia dos Yawalapití e em outubro de 2002 nas aldeias dos Mehináku e Waurá. Todos os índios com idade presumida de 20 anos ou mais eram chamados e identificados pelas fichas médicas em uso no trabalho de campo e introduzidas pelo Departamento de Medicina Preventiva da Universidade Federal de São Paulo (UNIFESP/EPM) desde os primórdios do programa de saúde que desenvolve no Parque Indígena do Xingu, ou seja, o final da década de 1960.

Amostras de sangue foram coletadas para dosagem de lipoproteínas séricas e ácido úrico, após jejum de dez horas. O colesterol total, suas frações e os triglicérides foram determinados por kits enzimáticos e processados em analisador automático. Os valores de LDL foram obtidos segundo as fórmulas de Friedwald et al. 11 . Para dosagem da glicose sangüínea utilizou-se glicosímetro e fitas Advantage (Roche, Estados Unidos).

Durante o exame físico foram tomadas, em duplicata, as medidas antropométricas por profissionais treinados. Uma balança antropométrica eletrônica portátil (modelo Línea, Kratos-Cas, São Paulo, Brasil) e um estadiômetro (modelo Microtoise Setup, CMS Weighing Equipment Ltd., Londres, Reino Unido) foram os instrumentos utilizados para a tomada do peso e da altura, respectivamente. Para a medição das circunferências corporais utilizou-se fita métrica (modelo 17, Grafco, São Paulo, Brasil). As circunferências do braço, cintura e quadril foram medidas, respectivamente, no ponto médio entre o acrômio e o olécrano, em torno da cicatriz umbilical e na região mais proeminente ao redor dos glúteos. Para a medição das dobras cutâneas utilizou-se o compasso de Lange (Lange Skinfold Caliper, 
Cambridge Scientific Industries, Cambridge, Reino Unido) e a técnica descrita por especialistas da Organização Mundial da Saúde (OMS) 12. As pressões arteriais sistólica (PAS) e diastólica (PAD) foram medidas usando-se, em rozídio, esfigmomanômetros aneróide (modelo Nova Presameter, Riester, Alemanha) e de mercúrio, para aferição concomitante. Após os indivíduos terem repousado por dez minutos, em posição sentada, foram feitas duas medições, sendo o valor final aquele que representou a média aritmética das medidas.

\section{Critério diagnóstico}

\section{- Quanto ao estado nutricional}

O índice de massa corporal (IMC), calculado como a razão entre o peso (em quilos) e o quadrado da altura (em metros), foi utilizado para classificação dos indivíduos quanto ao estado nutricional. Foram considerados como com sobrepeso e com obesidade os índios com valores de IMC entre 25 e $29,9 \mathrm{~kg} / \mathrm{m}^{2}$ e $30 \mathrm{~kg} / \mathrm{m}^{2}$, respectivamente 12 .

A medida da dobra cutânea triciptal foi utilizada com o objetivo de quantificar as reservas subcutâneas corporais de tecido adiposo; valores $>12 \mathrm{~mm}$, para homens e $>23 \mathrm{~mm}$, para mulheres, foram indicativos de excesso de tecido adiposo. A presença de obesidade abdominal foi caracterizada por valores de circunferência da cintura 80 ou $94 \mathrm{~cm}$, para mulheres e homens, respectivamente 12,13 .

\section{- Quanto à presença de doenças crônicas}

Para classificação dos indivíduos quanto à pressão arterial utilizou-se o critério proposto no relatório do Joint National Committee on Prevention, Detection, Evaluation, and Treatment of High Blood Pressure 14 para adultos maiores de 18 anos, a saber: normal se PAS $<120 \mathrm{mmHg}$ e $\mathrm{PAD}<80 \mathrm{mmHg}$, pré-hipertenso se PAS estiver entre $120-139 \mathrm{mmHg}$ e PAD entre $80-89 \mathrm{mmHg}$ e hipertenso quando os valores de PAS e PAD forem, respectivamente, $140 \mathrm{ou} 90 \mathrm{mmHg}$.

A presença de dislipidemia foi definida a partir de valores propostos pelo National Cholesterol Education Program Expert Panel 15, ou seja, níveis de colesterol total $>200 \mathrm{mg} / \mathrm{dL}$ ou triglicérides $>150 \mathrm{mg} / \mathrm{dL}$ ou LDL colesterol > 130mg/dL ou HDL colesterol $<40 \mathrm{mg} / \mathrm{dL}$. Considerou-se com hiperuricemia os indivíduos com valores de ácido úrico $>6$ ou $7 \mathrm{mg} / \mathrm{dL}$ para os sexos feminino e masculino, respectivamente.

Para a classificação dos indivíduos quanto ao grau de tolerância à glicose utilizou-se as reco- mendações da Sociedade Americana de Diabetes de 2003 16. Foram considerados normais os indivíduos com glicemia de jejum $<100 \mathrm{mg} / \mathrm{dL}$, com glicemia de jejum alterada os com valores de glicemia entre 100 e $125 \mathrm{mg} / \mathrm{dL}$ e com diabetes os com glicemia $126 \mathrm{mg} / \mathrm{dL}$.

\section{Análise estatística dos dados}

A existência de associações entre as presenças das doenças crônicas e as variáveis sexo e idade foi avaliada pela estatística qui-quadrado e pelas razões de prevalências de Mantel-Haenzsel (RP). Utilizou-se o teste t de Student para a comparação dos valores médios das variáveis biológicas dos indivíduos segundo sexo. O programa Stata (Stata Corporation, College Station, Estados Unidos) foi utilizado em todas as etapas da análise.

\section{Aspectos éticos}

O presente estudo encontra-se inserido no programa de saúde, assistência, ensino e pesquisa que a UNIFESP/EPM desenvolve no Parque Indígena do Xingu. A partir de seu inicio, em 1965, sempre houve preocupação em detectar mudanças no perfil de saúde-doença da população, tendo em vista o "surgimento" das chamadas doenças do mundo ocidental: obesidade, hipertensão arterial, dislipidemias e diabetes mellitus. $\mathrm{O}$ presente inquérito se insere nesse contexto. $\mathrm{Na}$ fase de planejamento do projeto foi feita explanação para as comunidades indígenas sobre os objetivos e procedimentos a serem adotados no estudo, ficando claro que a adesão e participação são voluntárias e que cada pessoa tem total liberdade para desistir a qualquer momento sem sofrer qualquer coerção ou dano, e sem deixar de receber a atenção da equipe médica para qualquer queixa que apresente. Os auxiliares de enfermagem indígenas e os agentes indígenas de saúde ajudaram a esclarecer a comunidade na língua nativa e participaram dos procedimentos. Os pesquisadores se comprometeram a enviar os resultados para a comunidade. Um termo de livre adesão foi dado para assinatura aos lideres e representantes dos povos estudados. Concomitantemente foi feita a gravação da leitura do referido termo e do pronunciamento das lideranças. $\mathrm{O}$ desenvolvimento do presente estudo foi aprovado pela Comissão Nacional de Ética em Pesquisa (CONEP) sob protocolo número 13.541. 


\section{Resultados}

Apresenta-se, na Tabela 1, a distribuição dos indivíduos segundo sexo e faixa etária. Observa-se que, em ambos os sexos, a maior proporção de indivíduos concentra-se nas faixas etária de 20 a 39 anos. Essa situação foi semelhante nas três tribos estudadas.

Índios das tribos Aruák do sexo masculino, quando comparados aos do sexo feminino, apresentaram valores médios menores de todas as dobras cutâneas e de HDL colesterol e maiores do índice de massa corporal, de circunferência do braço, de PAS e PAD, de triglicérides, de VLDL e de ácido úrico (Tabela 2).

As porcentagens de indivíduos segundo sexo, idade e a presença de doenças crônicas estão apresentadas na Tabela 3. Mulheres mais jovens (< 40 anos), quando comparadas aos homens, foram classificadas com menor freqüência como com dislipidemia $(52,8 \%$ vs. $90,2 \%)$ ou com alterações nos níveis pressóricos (21,8\% vs. 54,7\%), mas apresentaram maior prevalência de obesidade abdominal (76,4\% vs. 22,6\%). Situação semelhante foi observada entre os com idade maior ou igual a 40 anos, porém esta relação foi estatisticamente significante apenas para a presença de obesidade abdominal.

Na Tabela 4 estão apresentadas as razões entre as prevalências de alterações do estado nutricional e de algumas doenças crônicas segundo sexo, ajustadas para idade e tribo de origem. As prevalências de sobrepeso (51,8\%), obesidade (15\%), dislipidemia $(77,1 \%)$ e níveis pressóricos elevados $(37,7 \%)$ foram estatisticamente diferentes entre os gêneros. Entre aqueles do sexo masculino quando comparados às do feminino observaram-se prevalências estatisticamente maiores de excesso de peso ( $\mathrm{RP}=1,40$; IC95\%: 1,11-1,78 para sobrepeso e RP = 2,02; IC95\%: 1,16-3,53 para obesidade), de dislipidemia ( $\mathrm{RP}=1,42$; IC95\%: 1,17-1,71) sendo os níveis séricos diminuídos de HDL responsáveis, em ambos os sexos, por $82,2 \%$ dos casos de dislipidemia e alterações quanto à pressão arterial (RP = 2,87; IC95\%: 1,82-4,48 para pré-hipertensão e RP = 3,57; IC95\%: 0,99-12,89 para hipertensão). Vale destacar que dos 69 indivíduos com alguma alteração quanto à pressão arterial (pré-hipertensão ou hipertensão), 50\% eram da tribo Yawalapití. Já entre as mulheres, quando comparadas aos homens, observou-se maior prevalência de obesidade abdominal. Os valores das razões de prevalência mostram que essas relações permaneceram estatisticamente significantes mesmo após o ajuste para a idade e para as tribos. Nenhum caso de diabetes mellitus foi observado entre esses indivíduos.

\section{Discussão}

Em diversas partes do mundo, estudos com populações nativas despertam o interesse de pesquisadores especialmente pelo fato deles permitirem avaliar o impacto de mudanças sócio-econômicas e de estilo de vida sobre a saúde desses indivíduos. Estudos realizados há algumas décadas mostravam que, especialmente em relação aos fatores de risco cardiovasculares, esses povos, quando comparados aos caucasianos, apresentavam baixos riscos de doenças crônicas nãotransmissíveis 2,6,9,16. Contudo, principalmente em função das mudanças provocadas pelo processo de "ocidentalização", esses grupos populacionais sofreram rápida mudança no perfil de morbi-mortalidade, tornando-se mais susceptíveis às doenças cardiovasculares e ao diabetes mellitus, entre outras 1,2,4,16.

No Brasil, a transição epidemiológica ocorreu apresentando aspectos distintos daqueles observados na maioria dos países industrializados e mesmo em alguns latino-americanos como Chile e Cuba. Em nosso meio, observa-se superposição entre as etapas; a morbi-mortalidade persiste elevada tanto para as doenças crônicas não-transmissíveis quanto para as infecciosas e parasitárias 17 . Os achados do presente estudo, juntamente com os de outras publicações, 18,19 sugerem que esse processo também atinge a população indígena brasileira.

Em meados da década de 60, por ocasião da implantação do programa de assistência à saúde dos povos indígenas do Parque Indígena do Xingu, os profissionais da então denominada Escola Paulista de Medicina (UNIFESP/EPM) observaram que os níveis pressóricos da maioria dos indivíduos examinados eram normais e não sofriam alterações com o avançar da idade, ao contrário do que ocorre com populações não-índias; nessa ocasião a malária era a maior ameaça à saúde dos índios do Parque, atingindo adultos e crianças. Na população infantil, a malária era a principal causa de morte, superando os óbitos por infecções respiratórias agudas e por doenças diarréicas. Nos últimos anos houve considerável avanço no controle da malária no Parque Indígena do Xingu, com queda acentuada do número de casos registrados anualmente. A implantação de esquema de vacinação nos habitantes do Parque Indígena do Xingu ocorreu em 1966. Essa ação bloqueou a história de graves epidemias e levou ao crescimento dessa população. Apesar disso, no presente, ainda predominam as doenças infecciosas e parasitárias em relação às crônicas não-transmissíveis 18

De 1966 a 1971, em exame de 531 habitantes do Parque Indígena do Xingu, pertencentes às 
Número e porcentagem de índios Aruák (Mehináku, Waurá e Yawalapití) segundo sexo e idade. Alto Xingu, Brasil Central, 2000-2002

\begin{tabular}{|c|c|c|c|c|c|c|}
\hline \multirow{2}{*}{$\begin{array}{l}\text { Faixa } \\
\text { etária (anos) }\end{array}$} & \multicolumn{2}{|c|}{ Masculino } & \multicolumn{2}{|c|}{ Feminino } & \multicolumn{2}{|c|}{ Total } \\
\hline & $n$ & $\%$ & $\mathrm{n}$ & $\%$ & n & $\%$ \\
\hline $20-29$ & 39 & 38,2 & 34 & 34,3 & 73 & 36,3 \\
\hline $30-39$ & 20 & 19,6 & 26 & 26,2 & 46 & 22,9 \\
\hline $40-49$ & 17 & 16,7 & 17 & 17,2 & 34 & 16,9 \\
\hline $50-59$ & 14 & 13,7 & 15 & 15,2 & 29 & 14,4 \\
\hline $60 \mathrm{ou}+$ & 12 & 11,8 & 7 & 7,1 & 19 & 9,5 \\
\hline Total & 102 & 100,0 & 99 & 100,0 & 201 & 100,0 \\
\hline
\end{tabular}

Qui-quadrado $=2,43(p=0,657)$.

Tabela 2

Valores médios de variáveis metabólicas e antropométricas de índios Aruák segundo sexo. Alto Xingu, Brasil Central, 2000 2002.

\begin{tabular}{|c|c|c|c|}
\hline Variável & Masculino & Feminino & $\begin{array}{c}\text { Teste } \mathrm{t} \text { de } \\
\text { Student (valor de } \mathrm{p} \text { ) }\end{array}$ \\
\hline Idade (anos) & 39,0 & 38,4 & $0,31(0,380)$ \\
\hline Índice de massa corporal (kg/m2) & 26,9 & 25,6 & $2,56(0,006)$ \\
\hline Circunferência da cintura (cm) & 89,5 & 87,5 & $1,52(0,065)$ \\
\hline Razão cintura-quadril & 0,96 & 0,94 & $1,49(0,069)$ \\
\hline Circunferência do braço (cm) & 31,1 & 29,0 & $4,45(0,000)$ \\
\hline Prega cutânea triciptal (mm) & 10,2 & 16,5 & $9,14(0,000)$ \\
\hline Prega cutânea bicipital (mm) & 6,1 & 9,4 & $5,51(0,000)$ \\
\hline Prega cutânea subescapular (mm) & 14,4 & 19,0 & $5,00(0,000)$ \\
\hline Prega cutânea suprailíaca (mm) & 15,7 & 21,5 & $4,90(0,000)$ \\
\hline Pressão arterial diastólica (mmHg) & 74,8 & 68,2 & $5,15(0,000)$ \\
\hline Pressão arterial sistólica $(\mathrm{mmHg})$ & 116,7 & 107,3 & $4,78(0,000)$ \\
\hline Colesterol total (mg/dL) & 177,6 & 179,6 & $0,41(0,314)$ \\
\hline Triglicérides (mg/dL) & 148,6 & 125,0 & $2,19(0,015)$ \\
\hline HDL colesterol (mg/dL) & 32,8 & 41,3 & $6,98(0,000)$ \\
\hline LDL colesterol (mg/dL) & 115,3 & 113,3 & $0,50(0,309)$ \\
\hline VLDL colesterol (mg/dL) & 29,8 & 25,0 & $2,27(0,012)$ \\
\hline Ácido úrico (mg/dL) & 4,89 & 3,59 & $7,52(0,000)$ \\
\hline Glicemia de jejum (mg/dL) & 84,7 & 83,7 & $0,75(0,227)$ \\
\hline
\end{tabular}

famílias ou troncos lingüísticos Aruák, Karíb, Jê e Tupi, Baruzzi \& Franco 19 observaram que a prevalência de hipertensão arterial foi de $4 \%$ no sexo masculino, em níveis discretos, não ultrapassando $150 \mathrm{mmHg}$ a pressão sistólica e $90 \mathrm{mmHg}$ a diastólica; não foram observados casos de hipertensão arterial no sexo feminino. Os autores destacaram que grande parte dos índios examinados tinha massa muscular bem desenvolvida.
Em 1979, ao exame de 106 indígenas do Alto Xingu, área ao sul do Parque Indígena do Xingu, habitada por dez tribos, os níveis médios de colesterol e triglicérides séricos foram, respectivamente, de 154 e 91,4mg/dL (desvio padrão (dp) $=24$ e $36,4 \mathrm{mg} / \mathrm{dL}$ ) no sexo masculino e de $170 \mathrm{e}$ $92,1 \mathrm{mg} / \mathrm{dL}(\mathrm{dp}=26$ e $37,4 \mathrm{mg} / \mathrm{dL}$ ) no feminino. Não foram observadas alterações quanto a tolerância à glicose entre esses índios 19 . 
Porcentagem de índios Aruák segundo as presenças de alterações do estado nutricional, doenças crônicas, sexo e idade. Alto Xingu, Brasil Central, 2000-2002.

\begin{tabular}{|c|c|c|c|}
\hline Variável & Masculino & Feminino & $p^{*}$ \\
\hline \multicolumn{4}{|l|}{ 20-39 anos } \\
\hline \multicolumn{4}{|l|}{ Estado nutricional } \\
\hline Normal & 20,0 & 39,3 & 0,082 \\
\hline Sobrepeso & 61,8 & 48,2 & \\
\hline Obesidade & 18,2 & 12,5 & \\
\hline \multicolumn{4}{|l|}{ Obesidade abdominal } \\
\hline Sim & 22,6 & 76,4 & 0,000 \\
\hline Não & 77,4 & 23,6 & \\
\hline \multicolumn{4}{|l|}{ Excesso de tecido adiposo } \\
\hline Sim & 28,3 & 20,7 & 0,350 \\
\hline Não & 71,7 & 79,3 & \\
\hline \multicolumn{4}{|l|}{ Dislipidemia } \\
\hline Sim & 90,2 & 52,8 & 0,000 \\
\hline Não & 9,8 & 47,2 & \\
\hline \multicolumn{4}{|l|}{ Hipertensão arterial } \\
\hline Não & 45,3 & 78,2 & 0,001 \\
\hline Pré-hipertenso & 50,9 & 21,8 & \\
\hline Hipertenso & 3,8 & - & \\
\hline \multicolumn{4}{|l|}{ Hiperuricemia } \\
\hline Sim & - & 3,7 & 0,165 \\
\hline Não & 100,0 & 96,3 & \\
\hline \multicolumn{4}{|l|}{ Tolerância à glicose } \\
\hline Normal & 96,2 & 98,1 & 0,558 \\
\hline Glicemia de jejum alterada & 3,8 & 1,9 & \\
\hline Diabetes mellitus & - & - & \\
\hline \multicolumn{4}{|l|}{40 anos ou +} \\
\hline \multicolumn{4}{|l|}{ Estado nutricional } \\
\hline Normal & 30,2 & 46,1 & 0,251 \\
\hline Sobrepeso & 51,2 & 43,6 & \\
\hline Obesidade & 18,6 & 10,3 & \\
\hline \multicolumn{4}{|l|}{ Obesidade abdominal } \\
\hline Sim & 33,3 & 79,0 & 0,000 \\
\hline Não & 66,7 & 21,0 & \\
\hline \multicolumn{4}{|l|}{ Excesso de tecido adiposo } \\
\hline Sim & 21,4 & 10,5 & 0,187 \\
\hline Não & 78,6 & 89,5 & \\
\hline \multicolumn{4}{|l|}{ Dislipidemia } \\
\hline Sim & 91,2 & 81,2 & 0,222 \\
\hline Não & 8,8 & 18,8 & \\
\hline \multicolumn{4}{|l|}{ Hipertensão arterial } \\
\hline Não & 48,7 & 76,3 & 0,060 \\
\hline Pré-hipertenso & 40,5 & 15,8 & \\
\hline Hipertenso & 10,8 & 7,9 & \\
\hline \multicolumn{4}{|l|}{ Hiperuricemia } \\
\hline Sim & 2,9 & - & 0,293 \\
\hline Não & 96,3 & 100,0 & \\
\hline \multicolumn{4}{|l|}{ Tolerância à glicose } \\
\hline Normal & 94,1 & 91,9 & 0,714 \\
\hline Glicemia de jejum alterada & 5,9 & 8,1 & \\
\hline Diabetes mellitus & - & - & \\
\hline
\end{tabular}

* Qui-quadrado. 
Razões entre as prevalências de alterações do estado nutricional e de algumas doenças crônicas segundo sexo (masculino vs. feminino). Alto Xingu, Brasil Central, 2000-2002.

\begin{tabular}{|c|c|c|}
\hline Variável & $\mathrm{RP}$ * & IC95\% \\
\hline \multicolumn{3}{|l|}{ Estado nutricional } \\
\hline Normal & 1 & \\
\hline Sobrepeso & 1,40 & $1,11-1,78$ \\
\hline Obesidade & 2,02 & $1,16-3,53$ \\
\hline \multicolumn{3}{|l|}{ Obesidade abdominal } \\
\hline Sim & 0,39 & $0,28-0,54$ \\
\hline Não & 1 & \\
\hline \multicolumn{3}{|l|}{ Excesso de tecido adiposo } \\
\hline Sim & 1,43 & $0,83-2,46$ \\
\hline Não & 1 & \\
\hline \multicolumn{3}{|l|}{ Dislipidemia } \\
\hline Sim & 1,42 & $1,17-1,71$ \\
\hline Não & 1 & \\
\hline \multicolumn{3}{|l|}{ Hipertensão arterial } \\
\hline Não & 1 & 1 \\
\hline Pré-hipertenso & 2,87 & $1,82-4,48$ \\
\hline Hipertenso & 3,57 & $0,99-12,89$ \\
\hline \multicolumn{3}{|l|}{ Hiperuricemia } \\
\hline Sim & 0,54 & $0,04-6,40$ \\
\hline Não & 1 & \\
\hline \multicolumn{3}{|l|}{ Tolerância à glicose } \\
\hline Normal & 1 & \\
\hline Glicemia de jejum alterada & 0,88 & $0,22-3,51$ \\
\hline Diabetes mellitus & - & - \\
\hline
\end{tabular}

* Razão de prevalências de Mantel-Haenszel ajustadas para idade e tribo de origem.

Neste estudo, considerando todos os indivíduos simultaneamente, observou-se elevadas prevalências de excesso de peso $(51,8 \%$ e $15 \%$ de indivíduos com sobrepeso e obesidade, respectivamente), de obesidade abdominal (52,1\%), de dislipidemias $(77,1 \%)$ e de alterações quanto aos níveis pressóricos $(37,7 \%)$, indicando que estes sujeitos apresentam alto risco cardiovascular. Apesar do pequeno número de casos de glicemia de jejum alterada $(n=8 ; 4,6 \%)$, em estudos anteriores não se observaram casos de intolerância à glicose entre esses índios 9,19,20. Destaca-se que, excluindo-se a prevalência de obesidade abdominal, observou-se pior perfil metabólico entre os homens, independentemente da idade e da tribo de origem.

Apesar das limitações da comparação dos dados encontrados neste estudo com os de outros pesquisadores, pode-se afirmar que, nas últimas décadas, houve progressiva deterioração das condições de saúde desses indivíduos especialmente quando se consideram os resultados de outras pesquisas feitas com índios residentes no Parque Indígena do Xingu nas décadas de 60, 70 e 80, relatados por Pazzanese et al. 6, Baruzzi \& Franco 19 e Carvalho et al. ${ }^{9}$, nos quais casos de obesidade ou de outras alterações metabólicas (hipertensão, dislipidemia ou intolerância a glicose) foram raros ou completamente ausentes, sugerindo um possível efeito nocivo do maior contato destes com a sociedade nacional, responsável por mudanças no estilo de vida desses índios, tais como redução da atividade física (particularmente entre os homens) e do consumo de alimentos.

A alimentação dos índios do Alto Xingu, incluindo os Aruák, baseia-se principalmente no peixe, assado ou cozido, e na mandioca, na forma de beiju ou mingau, seguidos de outros tubérculos, amendoim, milho, banana, cana, mel e frutas silvestres. Em determinadas épocas do ano assume importância o consumo de ovos de tracajá, e do piqui, fruto de polpa amarela, rica em vitamina A. Eles não apreciam animais de caça 
com exceção de macacos e aves de maior porte como o mutum e o jaó. O sal tradicionalmente utilizado no Alto Xingu é extraído das cinzas do água-pé, planta aquática encontrada nos rios e lagoas da região; este sal é rico em potássio e pobre em sódio.

Em tempos mais recentes, observou-se o ingresso progressivo do sal comum, do açúcar e do óleo de cozinha (responsável pela ingestão freqüente de frituras). O consumo de outros alimentos não tradicionais como massas, bolachas e arroz, ocorre ocasionalmente. Os Yawalapití têm mais facilidades de acesso a esses alimentos do que os Mehináku e Waurá, o que poderia explicar, pelo menos em parte, a maior freqüência de alterações metabólicas entre esses indivíduos. Aparentemente, as mudanças observadas no hábito alimentar desses índios estariam concorrendo para o aumento do número de indivíduos com excesso de peso, bem como dos valores médios dos níveis pressóricos e de lípides séricos 18 .

O trabalho diário das mulheres índias praticamente não sofreu alterações; não se observam mudanças nas práticas agrícolas, que têm grande participação do sexo feminino. Já entre os homens, o uso de barcos a motor reduziu a freqüência de longas viagens fluviais em embarcações indígenas impulsionadas a remo, com conseqüente redução na atividade física habitual. Pode-se supor que essa situação contribuiu para a "substituição" de parte da massa muscular corporal por tecido adiposo refletida na elevada prevalência de excesso de peso (sobrepeso e obesidade) observada entre esses índios.

\section{Conclusão}

Neste estudo, as altas porcentagens de indivíduos com excesso de peso, dislipidemias e elevação dos níveis pressóricos, particularmente quando comparadas aos valores obtidos em estudos anteriores, revelam que esses índios pioraram seu perfil metabólico e "perderam a proteção" contra as doenças crônicas não-transmissíveis, quer pelo fato de outrora apresentarem biótipo "saudável" (menor porcentagem de tecido adiposo e maior de massa muscular), quer pela redução na prevalência de doenças infecciosas - principais causas de morbi-mortalidade, nas décadas passadas, entre esses povos. Apesar de ocorrer de maneira distinta daquela observada em outras partes do mundo, o processo de transição epidemiológica e nutricional observado em nosso meio (e também nas populações indígenas) tem em comum a consolidação do excesso de peso como um agravo nutricional associado à presença de diversas doenças crônicas não-transmissíveis. Esses achados sugerem a necessidade de implementação de medidas urgentes que visem tanto ao controle quanto à prevenção da obesidade e outros fatores de risco cardiovasculares também entre esses indivíduos.

\section{Resumo}

O objetivo deste trabalho foi descrever o perfil metabólico e antropométrico de índios Aruák (Mehináku, Waurá e Yawalapití) que habitam o Alto Xingu, Brasil Central. Em julho de 2000 e outubro de 2002, 201 indivíduos de ambos os sexos e com idade $\geq 20$ anos foram submetidos a exames físicos (antropometria e pressão arterial) e de laboratório (glicemia de jejum, lípides séricos e ácido úrico). Índios do sexo masculino, quando comparados aos do sexo feminino, apresentaram valores médios menores de dobras cutâneas e de HDL colesterol, e maiores do índice de massa corporal, de circunferência do braço, de pressão arterial sistólica e diastólica, de triglicérides, de VLDL e de ácido úri- co. As prevalências de sobrepeso (51,8\%,), obesidade (15\%), dislipidemia $(77,1 \%)$ e níveis pressóricos elevados $(37,7 \%)$ foram maiores entre os homens enquanto que as mulheres apresentaram maior prevalência de obesidade abdominal (52,1\%), independentemente da idade e da tribo de origem. Esses achados sugerem a necessidade de implementação de medidas que visem tanto ao controle como à prevenção da obesidade e outros fatores de risco cardiovasculares entre esses indivíduos.

Obesidade; Antropometria; Doença Crônica; Hipertensão; Índios Sul-Americanos 


\section{Colaboradores}

S. G. A. Gimeno participou do planejamento do estudo, análise estatística e redação do manuscrito. H. Pagliaro contribuiu com o planejamento do estudo e redação do manuscrito. R. G. Baruzzi participou da coleta dos dados, redação e revisão do manuscrito. D. Rodrigues contribuiu no planejamento do estudo e coleta dos dados. E. N. Conó e E. E. S. Lima participaram na coleta e organização dos dados.

\section{Agradecimentos}

Aos Mehináku, Waurá e Yawalapití, que possibilitaram a realização deste estudo e pela acolhida sempre amiga e cordial. Aos agentes indígenas de saúde e auxiliares de enfermagem indígenas pela colaboração no trabalho de campo. Ao Laboratório Central do Hospital São Paulo (UNIFESP/EPM) pela realização dos exames laboratoriais.

\section{Referências}

1. Liao Y, Tucker P, Okoro CA, Giles WH, Mokdad $\mathrm{AH}$, Harris VB, et al. REACH 2010 surveillance for health status in minority communities - United States - 2001-2002. MMWR Surveill Summ 2004; 53:1-36.

2. Story M, Stevens J, Himes J, Stone E, Rock BH, Ethelbak B, et al. Obesity in American-Indian children: prevalence, consequences and prevention. Prev Med 2003; 37(6 Pt 2):S3-12.

3. Gimeno SGA, Ferreira SRG, Franco LJ, Iunes M, Moisés RC. Prevalence and 7-year incidence of type 2 diabetes mellitus in a Japanese-Brazilian population: an alarming public health problem. Diabetologia 2002; 45:1635-8.

4. Cardoso AM, Mattos IE, Koifman RJ. Prevalência de fatores de risco para doenças cardiovasculares na população Guaraní-Mbyá do Estado do Rio de Janeiro. Cad Saúde Pública 2001; 17:345-54.

5. Santos RV, Coimbra Jr. CEA. Socioeconomic differentiation and body morphology in the Surui of Southwestern Amazon. Curr Anthropol 1996; 37:851-6.

6. Pazzanese D, Ramos OL, Lanfranchi W, Portugal O, Finatti AAC, Barreto HPCB, et al. Serum-lipid levels in Brazilian Indian population. Lancet 1964; 284:615-7.

7. Vieira-Filho JPB, Russo EMK, Juliano Y. As proteínas glicosiladas dos índios Parakategê. Arq Bras Endocrinol Metabol 1987; 31:33-4.

8. Nascimento JRL, Miranda RA, Xavier FB, Menezes RC. Hipertensäo arterial em índios adultos da tribo Tembé, nordeste do Estado do Pará. Rev Para Med 1998; 12:45-8.

9. Carvalho JJ, Baruzzi RG, Howard PF, Poulter N, Alpers MP, Franco LJ, et al. Blood pressure in four remote populations in the INTERSALT study. Hypertension 1989; 14:238-46.

10. von den Steinen K. Entre os aborígines do Brasil Central. São Paulo: Departamento de Cultura; 1940.

11. Friedwald WT, Levy RI, Friedrickson DS. Estimation of the concentration of low-density lipoprotein cholesterol in plasma, without the use of the preparative ultracentrifugate. Clin Chem 1972; 18:499-502.
12. World Health Organization. Physical status: the use and interpretation of anthropometry. Geneva: World Health Organization; 1995. (Technical Report Series, 854).

13. World Health Organization. Obesity: preventing and managing the global epidemic. Geneva: World Health Organization; 2000 (Technical Report Series, 894).

14. Chobanian AV, Bakris GL, Black HR, Cushman WC, Green LA, Izzo JL, et al. The Seventh report of the Joint National committee on Prevention, Detection, Evaluation and Treatment of High Blood Pressure: the JNC 7 report. JAMA 2003; 289: 2560-72.

15. National Cholesterol Education Program. Executive summary of the third report of the National Cholesterol Education Program (NCEP) expert panel on detection, evaluation, and treatment of high blood cholesterol in adults. JAMA 2001; 285:2486-97.

16. The Expert Committee on the Diagnosis and Classification of Diabetes Mellitus. Follow-up report on the diagnosis of diabetes mellitus. Diabetes Care 2003; 26:3160-7.

17. Schramm JMA, Oliveira AF, Leite IC, Valente JG, Gadelha AMJ, Portela MC, et al. Transição epidemiológica e o estudo de carga de doença no Brasil. Ciênc Saúde Coletiva 2004; 9:897-908.

18. Baruzzi R, Junqueira C. Parque Indígena do Xingu - saúde, cultura e história. São Paulo: Terra Virgem Editora; 2005.

19. Baruzzi R, Franco LJ. Amerindians of Brazil. In: Trowell HC, Burkitt DP, editors. Western diseases: their emergence and prevention. London: Edward Arnold Publishers; 1981. p. 138-53.

20. Coimbra Jr. CEA, Flowers NM, Salzano FM, Santos RV. The Xavánte in transition: health, ecology, and bioanthropology in Central Brazil. Ann Arbor: University of Michigan Press; 2004, p. 243-67.

Recebido em 08/Mar/2005

Versão final reapresentada em 06/Fev/2007 Aprovado em 23/Fev/2007 